\title{
Fundamental solutions of the complex Monge-Ampère equation
}

\author{
by Halil Ibrahim Celik and Evgeny A. Poletsky (Syracuse, N.Y.)
}

Abstract. We prove that any positive function on $\mathbb{C P}^{1}$ which is constant outside a countable $G_{\delta}$-set is the order function of a fundamental solution of the complex MongeAmpère equation on the unit ball in $\mathbb{C}^{\not \models}$ with a singularity at the origin.

1. The Monge-Ampère equation. Every locally bounded plurisubharmonic function $u$ on a domain $D \subset \mathbb{C}^{n}$ satisfies the Monge-Ampère equation $\left(d d^{\mathrm{c}} u\right)^{n}=\mu$, where

$$
d^{\mathrm{c}}=\frac{i}{2 \pi}(\bar{\partial}-\partial)
$$

and $\mu$ is a Borel measure on $D$ (see [2] and [7]). A definition of the Monge-Ampère operator for unbounded plurisubharmonic functions is not known. However, if a plurisubharmonic function $u$ is locally bounded away from its singularities, the Monge-Ampère operator can be defined as a positive Borel measure (see [6]). A fundamental solution at the origin of the Monge-Ampère equation on the unit ball $B$ in $\mathbb{C}^{n}$ is a plurisubharmonic function on $B$ such that $\lim _{|z| \rightarrow 1} u(z)=0$ and $\left(d d^{\mathrm{c}} u\right)^{n}=\alpha \delta_{0}$, where $\delta_{0}$ is the Dirac measure at 0 and $\alpha>0$. In this paper we consider only such fundamental solutions. For $n=1,2 \pi d d^{\mathrm{c}} u=\Delta u$ and, therefore, fundamental solutions of the Monge-Ampère equation are proportional to fundamental solutions of the Laplace equation which play very important role in classical potential theory. Their significance is based on the fact that fundamental solutions at a given point of the domain are proportional to each other. This implies the representation of subharmonic functions as convolutions of fundamental solutions and Laplacians.

1991 Mathematics Subject Classification: Primary 32F07, 32F05; Secondary 31C10.

Key words and phrases: plurisubharmonic functions, singularities, order function, Monge-Ampère equation. 
Therefore to represent plurisubharmonic functions as convolutions it is important to describe the set of fundamental solutions at some point. Examples of non-proportional fundamental solutions on domains in $\mathbb{C}^{2}$ are known and can be found in [1], [6, Ex. 5.7] and [8, Ex. 10.1]. However, all these examples have quite simple nature of singularities leaving some hope for complete description of fundamental solutions. In this paper we construct different fundamental solutions on $B \subset \mathbb{C}^{2}$ whose order functions are defined by arbitrary functions on arbitrary countable $G_{\delta}$-sets in the complex projective space $\mathbb{C P}^{1}$ (see Corollary 2.3 ). In our opinion it makes any description of such solutions too complicated to be useful.

Definition 1.1. A plurisubharmonic function on a domain $D$ in $\mathbb{C}^{n}$ is maximal if for every relatively compact open subset $G$ of $D$ and for each function $v$, upper semicontinuous on the closure $\bar{G}$ of $G$ and plurisubharmonic on $G, v \leq u$ in $G$ if $v \leq u$ on $\partial G$.

It was proved in [2] that a bounded plurisubharmonic function $u$ is maximal iff it satisfies the homogeneous Monge-Ampère equation $\left(d d^{\mathrm{c}} u\right)^{n}=0$. Following [7] we denote by $\operatorname{PSH}(B, 0)$ the set of plurisubharmonic functions on $B$, locally bounded on $B^{*}=B \backslash\{0\}$. Theorem 1.2 is a direct consequence of results in [6].

TheOrem 1.2. Let $u \in \operatorname{PSH}(B, 0)$ and $u(0)=-\infty$. Then $u$ is maximal on $B^{*}$ if and only if there exists a constant $\alpha>0$ such that $\left(d d^{\mathrm{c}} u\right)^{2}=\alpha \delta_{0}$.

It follows from this theorem that fundamental solutions are plurisubharmonic functions on $B$, maximal on $B^{*}$, and equal to 0 on $\partial B$.

We say that plurisubharmonic functions $u$ and $v$ on the ball are equivalent at $0(u \sim v)$ if their difference is locally bounded near the origin.

THEOREM 1.3. If fundamental solutions $u$ and $v$ are equivalent at 0 , then they are equal on $B$.

Proof. Since $u \sim v$ at 0 , there exist positive constants $c$ and $s$ such that $u(z)-c \leq v(z)$ for all $|z| \leq s$. For $|z|=r \leq s$, let $\delta=\delta(r) \geq-c / \log r$. Then $u(z)+\delta \log |z| \leq v(z)$ when $|z|=r$. This inequality is also true on $\partial B$. Hence by the maximality of $v$ on $B \backslash\{0\}, u(z)+\delta \log |z| \leq v(z)$ on the shell $R(r, 1)=\{z \in B: r<|z|<1\}$. We let $r \rightarrow 0$ to obtain $u(z) \leq v(z)$ on $B$. Similarly, $v(z) \leq u(z)$ on $B$ and therefore, $u=v$ on $B$.

To distinguish non-equivalent plurisubharmonic functions one can use the order function, which is defined as follows:

Definition 1.4. Let $u$ be a plurisubharmonic function on the unit ball $B$ in $\mathbb{C}^{n}$. The function $o_{u}$ defined as

$$
o_{u}(z)=\lim _{r \rightarrow 0} \inf _{|\gamma|=r} \frac{u(\gamma z)}{\log |\gamma z|},
$$


where $z \neq 0$ is a vector in $\mathbb{C}^{n}$ and $\gamma \in \mathbb{C}$, is called the order function of $u$ at the origin.

Since $o_{u}(z)=o_{u}(\gamma z), \gamma \neq 0$, we may assume that the order function is defined on the unit sphere $S$ in $\mathbb{C}^{n}$ or on the complex projective space $\mathbb{C P}^{n-1}$, which for $n=2$ coincides with the Riemann sphere $\overline{\mathbb{C}}$. If $u \in \operatorname{PSH}(B, 0)$ then $o_{u}(z)$ is bounded above. On the complex plane the order function is equal to $(2 \pi)^{-1} \Delta u(\{0\})$, where $\Delta u(\{0\})$ is the mass of the Laplacian of $u$ at the origin. Therefore,

$$
o_{u}(z)=(2 \pi)^{-1} \Delta_{\zeta} u_{z}(\{0\}),
$$

where $u_{z}(\zeta)=u(\zeta z)$ for $\zeta \in \mathbb{C}$.

It was proved in [3] that the order function is equal to the constant $l$ on $\mathbb{C} \mathbb{P}^{n-1}$ minus a pluripolar set and is greater than $l$ on this set. Now it follows immediately that if $u$ is negative on the unit ball, then $u(z) \leq l \log |z|$. It can be proved (see [4]) that $l$ is the Lelong number of $u$ at 0 . We do not need this observation, but we will use the name. It can be proved (see [5]) that the order function is a $G_{\delta}$-function, i.e., the sets $\left\{z: o_{u}(z) \geq \alpha\right\}$ are $G_{\delta}$ for all real $\alpha$.

Clearly equivalent plurisubharmonic functions have the same order function. The converse is not true as the example of maximal plurisubharmonic functions $u\left(z_{1}, z_{2}\right)=\max \left(\log \left|z_{1}\right|, 2 \log \left|z_{2}\right|\right)$ and $v\left(z_{1}, z_{2}\right)=\max \left(\log \left|z_{1}+z_{2}^{2}\right|\right.$, $\left.\log \left|z_{1}^{3}+z_{2}^{3}\right|\right)$ shows.

The following method allows us to construct fundamental solutions on $B$. Let $v$ be a negative plurisubharmonic function on $B$ such that $v(0)=-\infty$. Set

$$
v_{r}(z)= \begin{cases}0 & \text { if } z \in \bar{B} \backslash B_{r} \\ v(z) & \text { if } z \in B_{r}\end{cases}
$$

where $B_{r}$ is the ball of radius $r$. The function $v_{r}$ is upper semicontinuous. We set

$$
u_{r}(z)=\sup \left\{w: w(z) \text { is plurisubharmonic on } B \text { and } w \leq v_{r}\right\} .
$$

The functions $u_{r}$ are plurisubharmonic on $B$ and for $r_{1} \leq r_{2}, u_{r_{1}} \geq u_{r_{2}}$. Therefore, $\left\{u_{r}\right\}_{0 \leq r<1}$ is decreasing in $r$. Let $I^{\infty} v=\left(\lim _{r \rightarrow 0} u_{r}\right)^{*}$, where * denotes the upper semicontinuous regularization.

TheOREM 1.5. Let $v$ be a negative function in $\operatorname{PSH}(B, 0)$. If $I^{\infty} v(0)=$ $-\infty$, then $I^{\infty} v$ is a fundamental solution of the Monge-Ampère equation. Moreover, if $v$ is maximal on $B^{*}$, then $I^{\infty} v$ is equivalent to $v$ at the origin.

Proof. Since $u_{r} \leq 0$, the function $I^{\infty} v$ is plurisubharmonic on $B$. By Theorem 6.3 and Lemma 8.2 of [8], each function $u_{r}$ is maximal on $\{r<|z|<1\}$ and $\lim _{z \rightarrow \partial B} u_{r}(z)=0$. Moreover, by Choquet's topological lemma, $I^{\infty} v=\left(\lim _{j \rightarrow \infty} u_{r_{j}}\right)^{*}$, where $\left\{r_{j}\right\}$ is a monotonic sequence of positive numbers converging to 0 . Therefore, by Proposition 5.2 of [2], 
$I^{\infty} u$ is maximal on $B^{*}$ and, since $I^{\infty} v \geq u_{r}, \lim _{z \rightarrow \partial B} I^{\infty} v(z)=0$. Since $I^{\infty} v(0)=-\infty$, by Theorem 1.2, $I^{\infty} v$ is a fundamental solution of the Monge-Ampère equation.

Now suppose that $v$ is maximal on $B^{*}$. By the definition of $I^{\infty} v$ we have $I^{\infty} v \geq v$ near the origin. On the other hand, for any compact set $K \subset B$ containing the origin, there exists a smallest $t, 0<t<1$, such that $K \subset$ $B(0, t)=B(t)$, where $B(t)$ is the ball of radius $t$ and centered at $z=0$. Let $c=\inf _{S(t)} v(z)$, where $S(t)$ is the sphere of radius $t$. Then, since $v$ is locally bounded, $0>c>-\infty$. The function $v-c$ is maximal on $B^{*}$. For any $r$, $0<r<t$, we have $u_{r} \leq v-c$ on $B(t)$, which implies that $\lim _{r \rightarrow 0} u_{r}(z) \leq$ $v(z)-c$ on $B(t)$. Since the upper semicontinuous regularization does not change this inequality, it follows that $I^{\infty} v \leq v(z)-c$ on $B(t)$ and, therefore, $I^{\infty} v \sim v$ at the origin.

2. Construction of fundamental solutions. We need the following lemma.

Lemma 2.1. Let $A=\left\{a_{j}\right\}, j=1,2, \ldots$, be a countable $G_{\delta}$-set in $\mathbb{C}$ and $\left\{c_{j}\right\}$ be a sequence of positive real numbers. Then there are real numbers $\alpha_{j}>0$ such that:

(1) $\sum_{j=1}^{\infty} \alpha_{j}=1$;

(2) the series $\sum \alpha_{j} c_{j}$ converges;

(3) the function

$$
u(\zeta)=\sum_{j=1}^{\infty} \alpha_{j} \log \frac{\left|\zeta-a_{j}\right|}{\left|a_{j}\right|+j+1}
$$

is subharmonic on $\mathbb{C}$;

(4) $u(\zeta)=-\infty$ if and only if $\zeta \in A$;

(5) the functions

$$
u_{k}(\zeta)=\sum_{j \neq k} \alpha_{j} \log \frac{\left|\zeta-a_{j}\right|}{\left|a_{j}\right|+j+1}=-\infty
$$

if and only if $\zeta \in A \backslash\left\{a_{k}\right\}$.

Proof. Suppose that $A$ is the intersection of open sets $F_{i}$. Let $E_{1}=F_{1}^{\mathrm{c}}$ and $E_{j}=F_{j}^{\mathrm{c}} \cup\left\{a_{1}, \ldots, a_{j-1}\right\}$, where $F_{j}^{\mathrm{c}}$ is the complement of $F_{j}$ and $j \geq 2$. Choose $0<\alpha_{j}<2^{-j} \min \left\{1, c_{j}^{-1}\right\}$ such that the functions

$$
v_{j}(\zeta)=\alpha_{j} \log \frac{\left|\zeta-a_{j}\right|}{\left|a_{j}\right|+j+1}
$$

are less than $2^{-j}$ in absolute value on the set $G_{j}=E_{j} \cap \bar{B}(0, j)$. This is 
possible because this set is compact and does not contain $a_{j}$. Let

$$
u(\zeta)=\sum_{j=1}^{\infty} v_{j}(\zeta)
$$

If $\zeta \in A^{\mathrm{c}}$ then $\zeta$ belongs to all $G_{j}$ when $j$ is sufficiently large and, hence, the series for $u$ converges at $\zeta$. Moreover, $u(\zeta)>-\infty$. Since $v_{j}(\zeta)<0$ on $B(0, j), u$ is subharmonic on $\mathbb{C}$. Clearly $v\left(a_{j}\right)=-\infty$ for all $j$.

Let $\alpha=\sum \alpha_{j}$. If we replace $\alpha_{j}$ by $\alpha_{j} / \alpha$ in the definition of the function $v_{j}$, we get a subharmonic function $u$ on $\mathbb{C}$ satisfying conditions (1)-(4) of the lemma. To prove (5) we observe that $u_{k}\left(a_{j}\right)=-\infty$ when $j \neq k$ and $u_{k}(\zeta)>-\infty$ when $\zeta \notin A$. Since

$$
\left|v_{j}\left(a_{k}\right)\right| \leq \frac{1}{\alpha 2^{j}}
$$

when $j>k$, we see that $u_{k}\left(a_{k}\right)>-\infty$.

In the following theorem we construct a fundamental solution on $B$ with a given order function which is equal to a constant $c$ outside a countable $G_{\delta}$-set $L$ in $\overline{\mathbb{C}}$ and greater than $c$ on $L$.

TheOREM 2.2. Let $L$ be a set of lines $L_{j}=\left\{\left(z_{1}, z_{2}\right): a_{j} z_{1}+b_{j} z_{2}=0\right.$, $\left.\left|a_{j}\right|^{2}+\left|b_{j}\right|^{2}=1\right\}$ in $\mathbb{C}^{2}, j=1,2, \ldots$, such that its projection to $\overline{\mathbb{C}}$ is a $G_{\delta}$-set. Let $d, c, c_{1}, c_{2}, \ldots$ be real numbers satisfying $d>c_{j}>c>0$. Then there is a plurisubharmonic function $c \log |z| \geq u(z) \geq d \log |z|$ on $B$ such that $u$ is maximal on $B^{*}, o_{u}(z)=c_{j}$ if $z \in L_{j}$, and $o_{u}(z)=c$ if $z \notin L$.

Proof. Rotating coordinates if needed we may assume that all $a_{j} \neq 0$. We may also assume that $c=1$. The projection of $L$ into $\overline{\mathbb{C}}$ is the $G_{\delta^{-}}$ set $A=\left\{-b_{j} / a_{j}\right\}$. By Lemma 2.1 for this set we choose numbers $\alpha_{j}$ and functions $v_{j}$. We also require that

$$
\sum_{j=1}^{\infty} \alpha_{j} \log \frac{\left|a_{j}\right|}{\left|b_{j}\right|+(j+1)\left|a_{j}\right|}>-\infty .
$$

Let $z=\left(z_{1}, z_{2}\right), \widetilde{v}(z)=\sum_{j=1}^{\infty} u_{j}(z)$, where $u_{j}(z)=\max \left\{v_{j}^{\prime}(z), d_{j} \log |z|\right\}$,

$$
\begin{aligned}
v_{j}^{\prime}(z) & =\alpha_{j} \log \frac{\left|a_{j} z_{1}+b_{j} z_{2}\right|}{\left|b_{j}\right|+(j+1)\left|a_{j}\right|} \\
& = \begin{cases}v_{j}\left(\frac{z_{1}}{z_{2}}\right)+\alpha_{j} \log \left|z_{2}\right| & \text { if } z_{2} \neq 0, \\
\alpha_{j} \log \frac{\left|a_{j}\right|}{\left|b_{j}\right|+(j+1)\left|a_{j}\right|}+\alpha_{j} \log \left|z_{1}\right| & \text { if } z_{2}=0,\end{cases}
\end{aligned}
$$

and

$$
d_{j}=c_{j}-\sum_{k \neq j} \alpha_{k}=c_{j}-1+\alpha_{j}>\alpha_{j}
$$


Note that since $\left|a_{j} z_{1}+b_{j} z_{2}\right|<1$ on $B$, the functions $v_{j}^{\prime}$ are negative on $B$ and, therefore, $\widetilde{v}$ is either plurisubharmonic on $B$ or $\widetilde{v} \equiv-\infty$. Let $v(z)=$ $\max \{\widetilde{v}(z), d \log |z|\}$.

Let us find $o_{v}(z)$ when $|z|=1$. If $z \notin L$ and $z_{2} \neq 0$, then

$$
\widetilde{v}(\zeta z) \geq \sum_{j=1}^{\infty} v_{j}^{\prime}(\zeta z)=u\left(z_{1} / z_{2}\right)+\log \left|z_{2}\right|+\log |\zeta|,
$$

where $u$ is the function from Lemma 2.1 and $|\zeta|>0$. Since $u\left(z_{1} / z_{2}\right)>-\infty$, we see that $v(\zeta z)=\widetilde{v}(\zeta z)$ when $\zeta$ is small. If $z_{2}=0$ then

$$
\widetilde{v}(\zeta z) \geq \log |\zeta|+\sum_{j=1}^{\infty} \alpha_{j} \log \frac{\left|a_{j}\right|}{\left|b_{j}\right|+(j+1)\left|a_{j}\right|}>-\infty
$$

when $|\zeta|>0$, and again $v(\zeta z)=\widetilde{v}(\zeta z)$ when $\zeta$ is small. Moreover, $v_{j}^{\prime}(\zeta z)>d_{j} \log |\zeta|$ for every $j$ when $|\zeta|$ is smaller than some $\varepsilon_{j}>0$. Thus, $\Delta_{\zeta} u_{j}(\zeta z)(\{0\})=2 \pi \alpha_{j}$. Since $\widetilde{v}(\zeta z)>-\infty$ when $\zeta \neq 0$,

$$
\Delta_{\zeta} \widetilde{v}(\zeta z)(\{0\})=\sum_{j=1}^{\infty} \Delta_{\zeta} u_{j}(\zeta z)(\{0\})=2 \pi .
$$

So $o_{v}(z)=1$. Therefore, the Lelong number of $v$ is 1 and, since $v$ is negative, $v(z) \leq \log |z|$.

If $z \in L$, i.e., $z_{1} / z_{2}=-b_{j} / a_{j}$ for some $j$, then

$$
\begin{aligned}
\widetilde{v}(\zeta z) & \geq d_{j} \log |\zeta z|+\sum_{k \neq j}\left(v_{k}\left(\frac{z_{1}}{z_{2}}\right)+\alpha_{k} \log \left|\zeta z_{2}\right|\right) \\
& =c_{j} \log |\zeta|+\sum_{k \neq j}\left(v_{k}\left(\frac{z_{1}}{z_{2}}\right)+\alpha_{j} \log \left|z_{2}\right|\right)
\end{aligned}
$$

and again $v(\zeta z)=\widetilde{v}(\zeta z)$ when $\zeta$ is small. The same argument shows that $o_{v}(z)=c_{j}$ when $z \in L_{j}$.

Let $u=I^{\infty} v$. Note that $u(z) \geq v(z) \geq d \log (z)$. Since $\log |z|$ is maximal and $u_{r}(z) \leq \log |z|$ on $S_{r}=\{z:|z|=r\}$ and on $S$, we have $u_{r}(z) \leq \log |z|$ on $B \backslash B_{r}$. Hence, $u(z) \leq \log |z|$ and $u(0)=-\infty$. By Theorem 1.5, $u$ is a fundamental solution. Moreover, since $v(z) \leq u(z)$, we have $o_{u}(z)=1$ when $z \notin L$.

Let us prove that $o_{u}(z)=c_{k}$ when $z \in L_{k}$. There is a unitary change of coordinates such that in the new coordinates, which we will continue to denote by $z_{1}$ and $z_{2}$,

$$
v_{k}^{\prime}\left(z_{1}, z_{2}\right)=\alpha_{k} \log \frac{\left|z_{1}\right|}{\left|b_{k}\right|+(k+1)\left|a_{k}\right|}
$$

and $L_{k}=\left\{z_{1}=0\right\}$. Let $a=\left|b_{k}\right|+(k+1)\left|a_{k}\right|$ and $\beta=d_{k} / \alpha_{k}$. If $\left|z_{1}\right| \leq a\left|z_{2}\right|^{\beta}$ 
then

Now

$$
\max \left\{\alpha_{k} \log \frac{\left|z_{1}\right|}{a}, d_{k} \log |z|\right\} \leq d_{k} \log |z|
$$

$$
\widetilde{v}\left(z_{1}, z_{2}\right)=\max \left\{\alpha_{k} \log \frac{\left|z_{1}\right|}{a}, d_{k} \log |z|\right\}+\sum_{j \neq k} \max \left\{v_{j}^{\prime}\left(z_{1}, z_{2}\right), d_{j} \log |z|\right\} .
$$

The Lelong number of the sum in the expression above is equal to $1-\alpha_{k}$. Hence this sum does not exceed $\left(1-\alpha_{k}\right) \log |z|$. Therefore, $v(z) \leq c_{k} \log |z|$ when $\left|z_{1}\right| \leq a\left|z_{2}\right|^{\beta}$.

Take $m=[\beta]+2$, and for $c \in \mathbb{C},|c|<1$, consider the mappings

$$
g_{c}(\zeta)=2^{-1}\left(c \zeta^{m}, \zeta\right)
$$

of the unit disk $U$ into $B$. Let $A=\left\{w \in B: w=g_{c}(\zeta), \zeta, c \in U\right\}$. Clearly, $A \cap B^{*}$ is open and contains $L_{k} \cap B_{1 / 2}^{*}$. Since $\left|2^{-1} c \zeta^{m}\right| \leq a\left|2^{-1} \zeta\right|^{\beta}$ when $|\zeta| \leq a 2^{1-\beta}$, we see that the subharmonic function $v\left(g_{c}(\zeta)\right)$ on $U$ satisfies the inequality

when $|\zeta| \leq a 2^{1-\beta}$. Hence,

$$
v\left(g_{c}(\zeta)\right) \leq c_{k} \log |\zeta|
$$

$$
u_{r}\left(g_{c}(\zeta)\right) \leq c_{k} \log |\zeta| \leq c_{k}\left(\log \left|g_{c}(\zeta)\right|+\log 2\right)
$$

on $U$. Therefore, on $A$,

$$
\begin{aligned}
u_{r}(z) & \leq c_{k}(\log |z|+\log 2), \\
u(z) & \leq c_{k}(\log |z|+\log 2) .
\end{aligned}
$$

This proves that $o_{u}(z) \geq c_{k}$. But $u(z) \geq v(z)$, so $o_{u}(z)=c_{k}$.

This theorem implies the following corollary.

Corollary 2.3. Let $\varphi$ be a bounded function on $\mathbb{C P}^{1}=\overline{\mathbb{C}}$ which is equal to a constant $c>0$ outside a countable $G_{\delta}$-set $E$ and is greater than $c$ on $E$. Then there is a fundamental solution $u$ on $B$ such that its order function is equal to $\varphi$.

\section{References}

[1] E. Bedford and B. A. Taylor, The Dirichlet problem for a complex Monge-Ampère equation, Invent. Math. 37 (1976), 19-22.

[2] -, 一, A new capacity for plurisubharmonic functions, Acta Math. 149 (1982), 1-40.

[3] U. Cegrell and J. Thorbiörnson, Extremal plurisubharmonic functions, Ann. Polon. Math. 63 (1996), 63-69.

[4] H. I. Celik, Pointwise singularities of plurisubharmonic functions, Ph.D. Thesis, Syracuse University, 1996. 
[5] H. I. Celik and E. A. Poletsky, Order functions of plurisubharmonic functions, Studia Math. 124 (1997), 161-171.

[6] J. P. Demailly, Monge-Ampère operators, Lelong numbers and intersection theory, in: Complex Analysis and Geometry, Plenum Press, New York, 1993, 115-193.

[7] M. Klimek, Pluripotential Theory, Oxford University Press, New York, 1991.

[8] E. A. Poletsky, Holomorphic currents, Indiana Univ. Math. J. 42 (1993), 85-144.

Department of Mathematics

Syracuse University

Syracuse, New York 13244

U.S.A.

E-mail: hicelik@math.syr.edu

eapolets@mailbox.syr.edu

Reçu par la Rédaction le 26.7.1996

Révisé le 17.3.1997 